\title{
An AODV-based Clustering Approach for Efficient Routing in MANET
}

\author{
K. Tamizarasu \\ Asst. Prof. in CSE Department \\ Jayam College of Engineering and Technology \\ Dharmapuri, Tamil Nadu, India
}

\author{
M. Rajaram \\ Phd, Professor in EEE Department \\ Govt. College of Technology \\ Coimbatore, Tamilnadu, India
}

\begin{abstract}
In recent communication technologies and services the Mobile ad hoc network place an increasingly vital role, that dynamically establish the connection whenever required for the communication network resulting in a quickly changing in topology of the network and increase communication overheads. This dynamic topology, leads to significant routing overhead, scalability problems and battery power consumptions in MANETs. Consider this problem, in this paper a clustering approach have been proposed for ad hoc networks. In Clustering approach, the cluster head election is call upon for the constructing the path, reduce the communication over heads and scalability. For the path construction cluster-AODV routing protocol is applied and also the design goals of clustering algorithms are presented.
\end{abstract}

\section{General Terms}

Routing Protocols, Clusters.

\section{Keywords}

Mobile Ad hoc Networks (MANET), Cluster Management, AODV Clustering and Routing Schemes

\section{INTRODUCTION}

MANETs (Mobile Ad hoc Networks) is Wireless ad hoc networks and organize infrastructureless network. The main challenges of mobile ad hoc networking are Dynamic network topology and limited system resources. Many routing protocols were proposed for routing in MANETs [Chakeres, et al., 2004; Jiang, et al., 1999; Jacquet, et al., 2001]. In MANETs, when network's size exceeds a certain threshold decreases the performance, resulting in many routing algorithms performing only when network's size is small. To overcome bandwidth and battery power limitations, and reduce routing overhead, it is mandatory to make network organization smaller and manageable [Garcia, et al., 2003].

A clustering architecture provides solution for the Problem in MANET environments: network scalability, fault tolerance and reduction of communication overheads. Many present clustering algorithms use either geographical regions as clusters or form new ones proactively even when unnecessary [Denko, et al., 2003; Chiang, et al., 19997; Lin, et al., 1997]. An algorithm by Chatterjee, et al., [2000] creates clusters when necessary. But, this algorithm does not use routing protocol maintained information.

It is argued that if the routing algorithm is used to gather clustering information, clustering and routing overheads can be reduced greatly. AODV is a reactive routing protocol used in MANETs. Though AODV performs well with mobile nodes, it piles up high overheads with increased network size, nodal degree or number of communicating source-destination pairs. Through the use of AODV route construction and maintenance mechanisms, clustering architecture can be built when needed.

Clusters are maintained when data is to be forwarded. Such integrated routing and clustering scheme can improve throughput and reduce routing overhead. The main contributions of this paper include: (a) a clustering architecture based on extended AODV routing protocol for cluster formation, maintenance and purging work; and (b) an adaptive Cluster-AODV routing protocol using AODV and clustering information for expeditious route discovery, maintenance and packet delivery.

\section{RELATED WORK}

Two cluster head election algorithms have been proposed for mobile ad-hoc networks (MANET) that assume link steadiness, mobility, connectivity, cluster and weight are therefore closely related to our work.

Cluster Based Routing (CBR): In MANETS, the routing schemes are a major problem. Clustering Based Routing approach provides a solution for decrease routing control overhead and improve the network scalability. In CBR, group a node into clusters in each cluster one node act as a cluster heads in order to reduce the communications and control overheads. The major Design of Cluster Based Approach is to minimize on-demand route discovery traffic and use "local repair" to reduce route acquisition delay and new route discovery traffic.

Weighted Clustering Algorithm (WCA): The weighted distributed clustering algorithm takes into concern of ideal degree, broadcast power, battery power of mobile nodes, and mobility. Each node from the cluster acquires the weight values of all other nodes and by rebroadcasting gathers the information of other cluster heads. Within the region the node travels and it is not enclosed by any cluster head, for this the system calls upon the cluster set-up procedure.

\section{CLUSTER MANAGEMENT}

Clustering architecture provide network scalability, fault tolerance resulting in increased use of network resources. It could be used for resource management, routing and location management to lower communication and computational overheads. This section discusses cluster formation and maintenance 


\subsection{Clustering Algorithm Design Goal}

It is intended to integrate clustering with routing functions. The design aims of our clustering scheme include:

1. An algorithm using a routing protocol's control messages to form clusters with limited overhead.

2. An algorithm operating in localized and distributed manners and intertwining with nodes using only AODV.

3. The algorithm incurring limited cluster formation/maintenance overhead and supporting formation of on-demand clusters.

4. The algorithm minimizing network-wide flooding and being scalable.

The proposed scheme constructs/updates clustering architecture when clusters alone service is required. The ondemand nature of AODV's is utilized for the scheme. Nodes participating in clustering are known from $\mathrm{CHs}$ maintained topological information and individual nodes.

\subsection{Cluster Formation}

The aim of clustering is to ensure efficient use of network resources, enhance availability, reduce overheads and give scalable architecture [Gerla, et al., 1995; Jiang, et al., 1999; Garcai, et al., 2003]. The clustering algorithm choice affects clusters constancy. The proposed work divides a network into several two-hop clusters where in each cluster; a node can play one of five roles: cluster head, ordinary node, secondary cluster head, undecided node or gateway. A gateway is a node that communicates directly with more than two clusters.

A cluster head $(\mathrm{CH})$ is elected in every cluster and it is responsible for maintenance and inter and intra-cluster communication. A Secondary Cluster Head $(\mathrm{SCH})$ is also chosen to avoid the $\mathrm{CH}$ from transforming into a bottleneck [Lu, et al., 2004]. The SCH safely stores backup routing and cluster information, with the role being rotated among ordinary nodes. This election needs no extra overheads as any node wishing to serve as a $\mathrm{SCH}$ informs the $\mathrm{CH}$ and the latter informs cluster members. Also, every $\mathrm{CH}$ maintains two tables in addition to a routing table, an intra-cluster node table and a k-hop cluster table.

The intra-cluster node table has the IDs of cluster nodes. The k-hop table has the IDs of CHs of other clusters in a 2-hop neighborhood. The $\mathrm{CHs}$ coordinate every cluster and store shared information. All nodes regularly broadcast a hello message to ensure information about neighbors. To reduce the periodic broadcasting overhead, new/undecided nodes learn of their nearest $\mathrm{CH}$ on demand by forwarding a cluster head request packet. The nodes act as either ordinary nodes or a gateway based on present location.

\subsection{Cluster Head Election}

Many distributed algorithms were suggested for $\mathrm{CH}$ election in MANETs [Chiang, et al., 19997; Lin, et al., 1997]. Chiang, et al., revealed that the Lowest ID (LID) algorithm performed better than cluster head election algorithms based on Highest Connectivity (HC). The proposals in [Chatterjee, et al., 2000; Garcia, et al., 2003] use varied criteria for $\mathrm{CH}$ election. As cluster heads act as a responsible for route maintenance and intra and inter-cluster communication, they were expected to work for long durations when elected.
Nodal mobility and link failure are the chief reasons for cluster head re-election and changes in cluster membership.

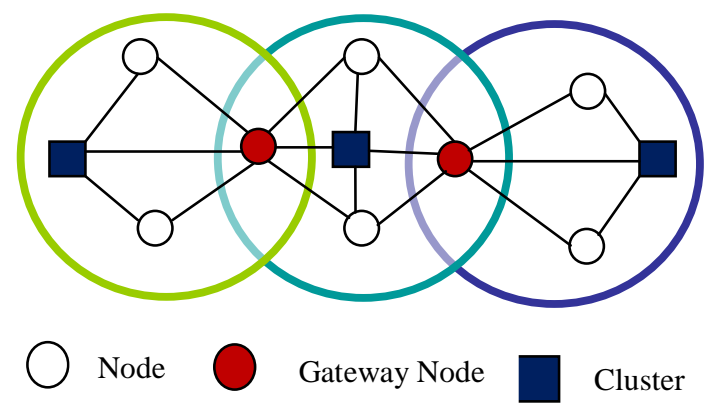

Fig 1: Cluster Head Election

Denko, et al., 2003, proposed a mobility-based clustering algorithm where a node is elected as cluster head only when the mobility index is below a specific threshold. This index is computed based on cluster membership and cluster head changes. When there is a tie, the lowest ID node is selected. In our cluster election algorithm, lowest ID clustering is first used to form clusters. Hence, a node is elected $\mathrm{CH}$ when it has the lowest ID. This forms the initial node configuration. Later, a node with a mobility index lower than its neighbors [Denko, et al., 2003] is used as criteria. In Figure 1, every node broadcasts mobility information to neighbors during a cluster head election. Once information is collected from neighbors every node checks to see if it is the node with the lowest mobility index. Once this confirmed it becomes a cluster head itself and informs all neighbors.

\subsection{Cluster Maintenance}

Cluster maintenance consists of two parts: intra-cluster and inter cluster maintenance.

\subsubsection{Intra-cluster maintenance}

To keep the neighbor table and $\mathrm{CH}$ information regular, nodes exchange hello messages at periodic intervals. A hello message has information about a node's ID and roles. If no hello message is received from a neighbor during the ALLOW_HELLO_LOST interval, the neighbor is thought lost and removed from the table. An ordinary node checks the neighbor table to check whether the $\mathrm{CH}$ is still alive. When no $\mathrm{CH}$ is found to exist, a new $\mathrm{CH}$ is elected in the neighborhood. Local maintenance is carried out, when a $\mathrm{CH}$ fails.

\subsubsection{Inter-cluster maintenance}

Every cluster head has a K-hop cluster table, containing all network k-hop $\mathrm{CHs}$ alive. Each $\mathrm{CH}$ then informs neighboring $\mathrm{CHs}$ that it is alive by forwarding a HeadAlive message. For example, $\mathrm{CH} 1$ receives a HeadAlive message from $\mathrm{CH} 2$. If $\mathrm{CH} 1$ discovers that $\mathrm{CH} 2$ exists in the $\mathrm{CH}$ table, $\mathrm{CH} 2$ 's expiration time is updated. Otherwise, a new $\mathrm{CH}$ entry for $\mathrm{CH} 2$ could be inserted and its expiration time would be set by improving $\mathrm{CH}$ update time to the present. When no HeadAlive message is received from cluster heads during a HEAD_UPDATE_INTERVAL interval, such a cluster is though unavailable. When no HeadAlive message is received during ALLOW_HEADALIVE_LOST interval, that $\mathrm{CH}$ is considered unavailable and removed from the $\mathrm{CH}$ table 


\subsection{Implementation of the AODV Clustering and Routing Schemes}

A K-hop $\mathrm{CH}$ table maintains $\mathrm{K}$-hop neighbor cluster information. We used $\mathrm{k}=2$, where only two hop clusters are considered. The K-hop $\mathrm{CH}$ table maintains fields such as $\mathrm{CH}$ ID, $\mathrm{CH}$ status, cluster size, $\mathrm{CH}$ expiration time and number of times the hello message has been lost. The CH's expiration time is the current time plus the HeadAliveUpdate interval. A CH's status could be either "alive" or "not alive." A CH's status will be marked as "not alive" when its HeadAlive message is not received by other $\mathrm{CHs}$ within its expiration time. A CH entry will be removed from a $\mathrm{K}$-hop $\mathrm{CH}$ table if the number of times that the HeadAlive message has not been received exceeds three. In the following section we will present the various data structure added to the OADV to implement the proposed clustering architecture.

\subsubsection{Hello Message and Neighborhood Maintenance}

Prior to a hello message being forwarded the sender node adds status information to it. The hello message sender can be a $\mathrm{CH}$, a gateway, ordinary node or undecided node. The hello message's extension includes the following: source address, lifetime and current status.

A neighbor table stores the neighbor's ID, expiration time, status and the many times a hello message was lost. When a node is in receipt of a hello message from neighbors, it checks whether the neighbor is alive in the neighbor table. If existence is confirmed, the neighbor's expiration time is updated. Or else a new entry is added. When anode has not received a hello message from another in three subsequent hello intervals, it is thought to be unconnected and is removed from the neighbor table.

\subsubsection{Cluster Management Module}

The following three modules are used to implement an AODV based clustering algorithm.

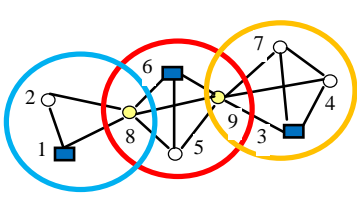

(a)

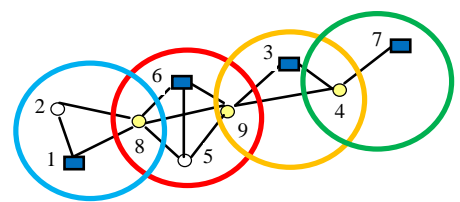

(c)

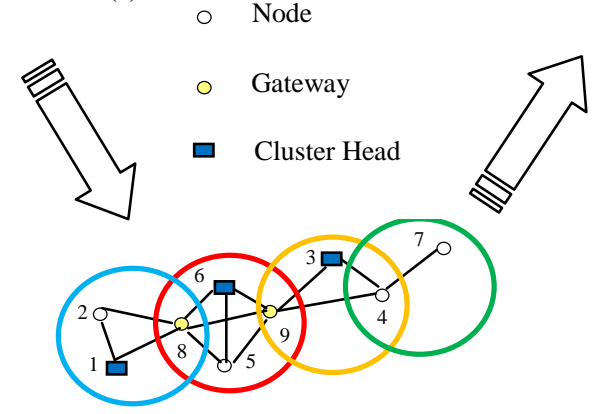

(b)

Fig 2: Cluster re-election when no cluster exists in the neighbor table

\subsubsection{Cluster head election module.}

A node invokes a Cluster Head Election module to elect cluster heads when there is no $\mathrm{CH}$ or when multiple $\mathrm{CH}$ are in proximity to each other. The procedure involves the following steps:
1. A node checks whether it has the lowest mobility index among non-gateway neighbors. It changes status to $\mathrm{CH}$ and broadcasts this to neighbors;

2. When it has the lowest mobility index, it declares itself $\mathrm{CH}$ and notifies other nodes. Otherwise, it changes its status to ordinary node.

Clusters are monitored to prevent formation of multiple $\mathrm{CHs}$ and also the absence of a $\mathrm{CH}$ in a cluster. This is illustrated through examples. Figure 2(a) reveals the topology of a hypothetical clustered-network at time $t$. In Figure 2(a), nodes 1, 3 and 6 are CHs. Nodes 8 and 9 are gateways while nodes $2,4,5$ and 7 are ordinary nodes. Assuming that at time $t 2$ the network's topology changes to Figure 2(b), where node 7 has moved from its original cluster. When node 7 checks the neighbor table, it cannot locate a cluster head. Thus node 7 uses the Cluster Head Election module to elect a new $\mathrm{CH}$ and the result is shown in Figure 2(c), where node 7 becomes a new $\mathrm{CH}$ and node 4 the new gateway.

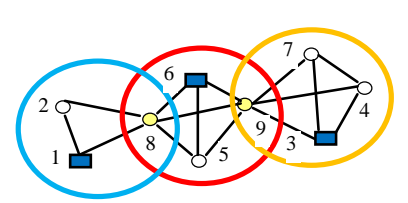

(a)

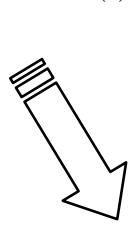

- Node

- Gateway

$\square \quad$ Cluster Head

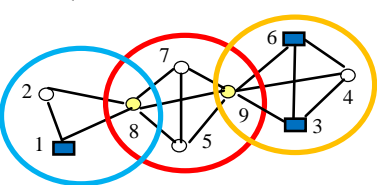

(b)

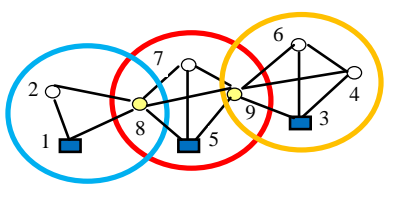

(c)

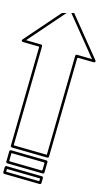

Fig 3: Cluster re-election when more than one cluster head exists

Figure 3 reveals shows a situation where many $\mathrm{CHs}$ exist in one cluster as shown in (a). When the network's topology changes from Figure 3(a) to Figure 3(b) at the time $t 2$, nodes 6 and 7 exchange positions. Nodes 6 and 3 find two $\mathrm{CHs}$ among their neighbors, as shown in Figure 3(b). Then both nodes 3 and 6 invoke the Cluster Head Election Module to select a new cluster head. After cluster election, node 6 throws away its $\mathrm{CH}$ status and changes itself into an ordinary node. Node 3 remains a $\mathrm{CH}$. Again, the cluster with nodes 5, 7, 8 and 9 elect node 5 as the new $\mathrm{CH}$. Network topology after the $\mathrm{CH}$ reelection is seen in Figure 3(c).

\section{CLUSTER-AODV-BASED ROUTING}

The AODV protocol sends many packets in comparison to other reactive protocols like DSR. So, when network's size increases, node degree also increases proportionately, leading to congestion in the network. Clustering reduces this through localized route discovery and maintenance. The suggested Cluster AODV scheme uses clustering architecture and AODV functionalities for routing. This section discusses mechanisms used by Cluster-AODV to lower routing overhead and allows scalability while ensuring good packet delivery ratio. 


\subsection{Intra-cluster routing.}

Intra-cluster routing is routing within a cluster. Each node has routing information on its cluster. When a node lacks a route to a destination that is in the cluster it sends a Local Route Request (LRREQ). When route failures ensure lack of reply to a RREP, local route maintenance is undertaken within a cluster.

\subsection{Inter-cluster routing.}

Inter-cluster routing is routing among clusters. The $\mathrm{CH}$ has a 2-hop cluster topology also maintained in a $\mathrm{SCH}$ to minimize one point of failure. When routes cannot be found in a cluster once a LRREQ message has been issued, a $\mathrm{CH}$ uses a RREQ message to locate a destination via a gateway to 2-hop neighbor clusters. To lower RREQ flooding packet overhead only gateways and CHs forward the RREQ. Ordinary nodes are not involved in RREQ packets in inter-cluster communication.

\subsection{Route maintenance.}

Similar to route maintenance, to cluster maintenance starts when a route fails within a cluster and is re-constructed locally using LRREQ and RREQ with 2-hop topology information. When LRREQ fails, an AODV procedure is used and the usual RERR is forwarded to source nodes for route reconstruction. The source node follows the same process to repair failed routes, first locally and then others.

The processes involving a new node which joins and an existing node leaving are carried out based on hello messages from AODV.. When CHs exchange neighborhood information with cluster members, a new node close by can register with a $\mathrm{CH}$ by using a RREQ message. A node acts as gateway when it registers with two $\mathrm{CHs}$. When a node goes away from the present $\mathrm{CH}$, it switches its role to that of an ordinary node, a gateway or will be undecided. It will be erased from the old $\mathrm{CH}$ and old members' routing entries are updated accordingly.

\section{EXPERIMENTAL SETUP}

The OPNET modeler 14.5 simulation tool is used to evaluate performance. When simulation started, 100 nodes were placed randomly within a $1000 \mathrm{~m} \times 1000 \mathrm{~m}$ area and the transmission range was fixed at $250 \mathrm{~m}$. The random waypoint mobility model [Broch, et al., 1998] was used to simulate mobility with a 40 second pause time. Other simulation parameters are seen in Table 1 .

Table 1. Simulation Parameters

\begin{tabular}{|l|l|}
\hline Parameter & Default Values \\
\hline Node Speed $(\mathrm{m} / \mathrm{sec})$ & $0-20$ \\
\hline Transmission rate & 4 pkts/sec \\
\hline Traffic Model & CBR, 30 sources \\
\hline Packet Size & 512 bytes \\
\hline Simulation Time $(\mathrm{sec})$ & 1200 \\
\hline
\end{tabular}

The proposed AODV Clustering approach is evaluated for throughput, delay and number of hops.

\subsection{Results and Discussion}

The experiments were conducted based on the above simulation parameters. Each data point in the graph represents an average of ten simulation runs. Figure 4 shows the throughput in bits/sec for the proposed AODV based clustering protocol. Figure 5 show the delay in MANET.

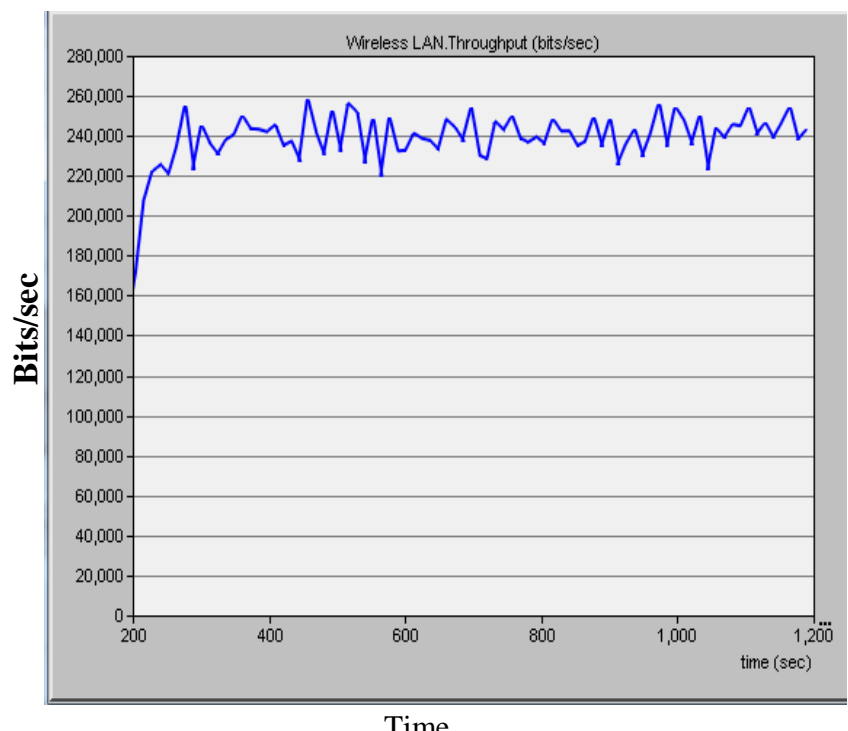

Fig 4: Throughput for the AODV based clustering protocol

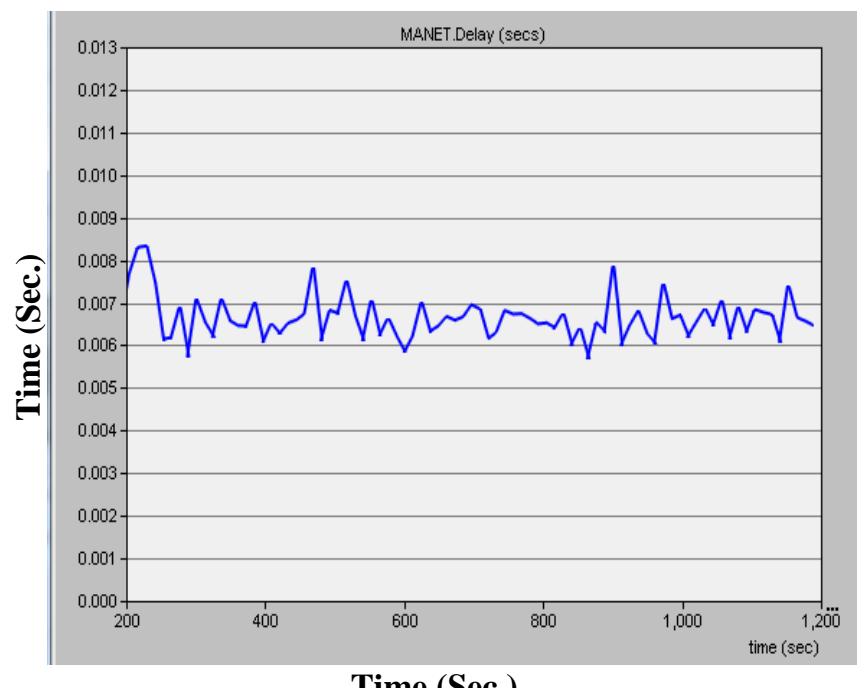

Time (Sec.)

Fig 5: Delay in MANET

\section{CONCLUSIONS}

This paper presents an AODV-based clustering and routing scheme for MANETs. The scheme is used for integrated routing and message delivery in clustered networks. The proposed clustering architecture was evaluated using simulation experiments. The simulation results show that the algorithm builds stable clusters with low communication overhead due to its localized, distributed and reactive nature. 


\section{REFERENCES}

[1] Chakeres I.D. and Belding-Royer E.M, AODV Routing Protocol Implementation Design, Proceedings of the International Workshop on Wireless Ad hoc Networking (WWAN), Tokyo, Japan, March 2004, pp. 698-703.

[2] Chatterjee M, Das S. K and Turgut D, An On-Demand Weighted Clustering Algorithm (WCA) for Ad-Hoc Networks, Proceedings of IEEE GLOBECOM 2000.

[3] Chiang C.C, Wu H.K, Liu W and Gerla M, Routing in Clustered Multihop, Mobile Wireless Networks With Fading Channel, Proceedings of IEEE Singapore International Conference on Networks SICON'97, pages 197-211, Singapore, Apr. 14-17, 1997.

[4] David A.; David B. Johnson; Josh Broch; Maltz; Jorjeta Jetcheva.;Yih-Chun Hu; A performance comparison of multi-hop wireless ad hoc network routing protocols. In Mobicom 1998, pages 85-97.ACM, 1998.

[5] Denko M.K, Analysis of Clustering Algorithms in Mobile Ad Hoc Networks, Proceedings of International Conf. on Wireless Networks, pp. 98-105, 2003.

[6] Gerla M and Tsai J, Multicluster, Mobile, Multimedia Radio Network, Wireless Networks, vol. 1, no. 3, 1995, pp. 255-65.

[7] Jacquet P, Muhlethaler P, Qayyum A, Laouiti A, Viennot L, and Clausen T., Optimized Link State Routing Protocol Internet Draft, draft-ietf-manet-olsr-04.txt, work in progress, June 2001.
[8] Jiang M., LI J., and Tay Y. C., Cluster Based Routing Protocol (CBRP) Functional Specification Internet GDraft, draft- ietf-manet-cbrp.txt, work in progress, June 1999.

[9] Kamvar S.D, Schlosser M.T, and Garcia-Molina H, The Eigen Trust Algorithm for reputation management in P2P networks, in: Proceedings of the Twelfth International World Wide Web Conference, Budapest, Hungary, 2003, pp. 640-651.

[10] Lu H and Denko M. K, Reliable Data Storage and Dissemination in Mobile Ad hoc Network, Proceedings of International workshop on Theoretical and Algorithmic Aspects of Wireless Ad Hoc, Sensor and Peer-to-Peer Networks, June 2004, Chicago, USA.

[11] Chatterjee M, Das S and Turgut D, "WCA: a weighted clustering algorithm for mobile ad hoc networks," Journal of Cluster Computing (Special Issue on Mobile Ad hoc Networks), 5, pp.193-204, 2002.

[12] Brust M.R, Andronache A and Rothkugel S, "WACA: A Hierarchical Weighted Clustering Algorithm optimized for Mobile Hybrid Networks," Proceedings of the Third International Conference on Wireless and Mobile Communications (ICWMC'07), 2007.

[13] Ramachandran L, Kapoor M, Sarkar A and Aggarwal A, "Clustering algorithms for wireless ad hoc networks," In Proceeding: Workshop on Discrete Algorithms and Methods for Mobile Computing and Communications, Boston, pp. 54-63, 2000. 